\title{
Establishment of a Primary Culture System of Human Periodontal Ligament Cells that Differentiate into Cementum Protein 1-expressing Cementoblast-like Cells
}

\author{
HIROSHI KADOKURA ${ }^{1}$, TAKAHIDE YAMAZAKI ${ }^{1}$, YOSHIKO MASUDA ${ }^{1}$, YUKA KATO ${ }^{1}$, \\ AKIHIKO HASEGAWA ${ }^{2}$, HIROSHI SAKAGAMI ${ }^{3}$ and SATOSHI YOKOSE ${ }^{1}$ \\ ${ }^{1}$ Division of Endodontics and Operative Dentistry, Department of Restorative and Biomaterial Sciences, \\ Meikai University School of Dentistry, Saitama, Japan; \\ ${ }^{2}$ Division of Internal Medicine, Department of Comprehensive Medical Sciences, \\ Meikai University School of Dentistry, Saitama, Japan; \\ ${ }^{3}$ Meikai University Research Institute of Odontology (M-RIO), Saitama, Japan
}

\begin{abstract}
Background/Aim: A better understanding of cementogenesis and cementoblast differentiation would be useful for periodontal therapy. The aim of this study was to establish a cell culture system that reflects cementum formation in periodontal tissue and determine whether or not isolated and cultured primary human periodontal ligament (PDL) cells could be used for the study of the differentiation of cementoblast. Materials and Methods: PDL cells were isolated from the outgrowths of tissue fragments of human PDL. PDL cells were incubated for up to 21 days in differentiation medium containing $\beta$-glycerophosphate and ascorbic acid. The changes in the cells were detected by alkaline phosphatase $(A L P)$ and von Kossa staining. Real-time polymerase chain reaction was also performed for cementum protein 1 (CEMP1), which is a specific marker of cementoblasts and their progenitors. Results: On day 5, a small number of PDL cells, which were fibrous, were positive for ALP. On day 7, almost all cells were positive for ALP. On day 14, mineralization nodules appeared, as seen by positive von Kossa staining; the nodules increased in number and size by day 21. The expression of CEMP1 was detected on day 5 , and its expression level increased gradually by day 7 , reached a peak on day 14, and decreased by day 21 . Conclusion: Human PDL cells were used to establish a culture system that reflects cementum formation. Our results suggested
\end{abstract}

This article is freely accessible online.

Correspondence to: Hiroshi Kadokura, DDS, Ph.D., Division of Endodontics and Operative Dentistry, Department of Restorative and Biomaterial Sciences, Meikai University School of Dentistry, 1-1, Keyakidai, Sakado, Saitama, 350-0283, Japan. Tel: +81 492855511, Fax: +81 492878073, e-mall: h-kadokura@dent.meikai.ac.jp

Key Words: Periodontal ligament cells, cementoblasts, CEMP1. that this culture method is convenient and useful for the study of cementogenesis and cementoblast differentiation.

Periodontal ligament (PDL) tissues contain periodontal stem cells, which can differentiate into cementoblast-like cells, adipocytes, and osteoblast-like cells (1-4). A better understanding of cementogenesis and the differentiation of periodontal stem cells into cementoblasts would be useful for periodontal therapy $(5,6)$. A previous study showed that the expression of cementum protein 1 (CEMP1) is limited to cementoblasts, a subpopulation of PDL cells, and to cells located around vessels in the periodontium (7). This localization indicates that CEMP1 is a marker of cementoblasts and their progenitors (8).

The aim of this study was to establish a culture system of PDL cells that can differentiate into CEMPl-expressing cementoblast-like cells. A primary culture of human PDL cells isolated from the root of a tooth, that was extracted for orthodontic treatment, was prepared. Histochemical staining for alkaline phosphatase (ALP) and von Kossa stainings were performed on the cells, to detect mineralized nodules. The expression of $C E M P 1$ by the cells was examined using real-time polymerase chain reaction (PCR).

\section{Materials and Methods}

Cell culture. This study was approved by the Ethics committee of the Meikai University School of Dentistry (A1616). The human tooth that was used in this study was extracted for orthodontic treatment. After extraction, the tooth was immediately infused with calcium- and magnesium-free phosphate-buffered saline (Wako Pure Chemical Industries, Osaka, Japan). Human PDL tissue fragments were scraped from the root surface with an excavator. The tissue fragments were cultured in alpha modification of Eagle's medium ( $\alpha$ MEM; Gibco, Grand Island, NY, USA) supplemented with $10 \%$ bovine serum (Gibco), $100 \mathrm{U} / \mathrm{ml}$ penicillin, and $100 \mu \mathrm{l} / \mathrm{ml}$ streptomycin (Gibco) in 
a $10-\mathrm{cm}$ dish (Falcon, Corning, NY, USA) at $37^{\circ} \mathrm{C}$ and $5 \% \mathrm{CO}_{2}$. The medium was changed every other day. The PDL cells were obtained from the outgrowths of the tissue fragments. After reaching a subconfluent state, the cells were detached from the dish with a trypsinEDTA enzyme solution (Gibco), collected and centrifuged at $130 \times g$ for $5 \mathrm{~min}$. The cells were resuspended in $\alpha$ MEM, and incubated in a 16-cm dish (Falcon) under the same conditions described for the 10$\mathrm{cm}$ dish. After reaching a sub-confluent state in the 16-cm dish, the cells were detached from the dish using the trypsin-EDTA enzyme solution (Gibco), and were collected and centrifuged at $130 \times g$ for 5 min. Single-cell suspensions were obtained by passing the cells through a $40-\mu \mathrm{m}$ strainer (Falcon). To obtain a large number of cells, the cells were cultured for three passages in $16-\mathrm{cm}$ culture dishes (Falcon). The cells of the third passage were used for all experiments in this study. The cells were seeded into 6-multi-well plates (Falcon) at a density of $2 \times 104 / \mathrm{cm}^{2}$ and cultured in differentiation inducing medium which is $\alpha$ MEM supplemented with $10 \%$ bovine serum, 1 $\mathrm{mM} \beta$-glycerophosphate (Wako Pure Chemical Industries), $50 \mu \mathrm{m} / \mathrm{ml}$ ascorbic acid (Wako Pure Chemical Industries), $100 \mathrm{U} / \mathrm{ml}$ penicillin, and $100 \mu \mathrm{l} / \mathrm{ml}$ streptomycin. The medium was changed every other day, and the cells were incubated for up to 21 days.

ALP staining and von Kossa staining. The cells were fixed with $10 \%$ neutral formalin (Wako Pure Chemical Industries) for $5 \mathrm{~min}$ at room temperature, then washed in Tris-Buffered Saline (Takara Bio Inc., Shiga, Japan) three times. The localization of ALP was determined by using naphthol AS-MX phosphate (Sigma Chemical Co., St. Louis, MO, USA) and fast blue BB salt (Wako Pure Chemical Industries). After determining the localization of ALP in the cells, von Kossa staining was performed to detect mineralization nodules formed by the cells. von Kossa staining was performed according to the method of Yokose et al. (9).

Real-time PCR. The total RNA was extracted from the cultured cells on days $5,7,14$, and 21 from the 6-multi-well plates by using RNAiso Plus (Takara Bio Inc.) according to the manufacturer's protocol. One microgram of each total RNA was converted into cDNA by reverse transcriptase (Takara Bio Inc.). Real-time PCR reactions were carried out using Thermal Cycler Dice (Takara Bio Inc.), and were performed in $12.5 \mu \mathrm{l}$ of solution containing cDNA, forward and reverse primers, and SYBR Green Real-time PCR Master Mix (Takara Bio Inc.); the mRNA levels were normalized to the mRNA levels of a housekeeping gene, GAPDH. The primer pairs used were as follows: forward, 5'CATACTGCCCTCCAGCACCA-3', reverse, 5'-TGAGTGCCACGG ATGACCA-3' for human CEMP1 (Takara Bio Inc.); and forward, 5'GCA CCGTCAAGGCTGAGAAAC-3', reverse, 5'-TGGTGAAGA CGCCAGTGGA-3' for GAPDH (Takara Bio Inc.). GAPDH was used as an internal control, and the $2^{-\Delta \Delta C t}$ method was applied to determine the differences in relative expression.

Statistical analyses. The results are expressed as the means \pm standard deviation (SD). Statistical analyses between two groups were performed with the Mann-Whitney $U$-test, and those between multiple groups was performed with the Kruskal-Wallis $\mathrm{H}$ test.

\section{Results}

ALP staining and von Kossa staining. The human PDL cells used in this study reached confluence on day 2 , and a small number of the cells, which were fibrous, were positive for
ALP on day 5 (Figure 1a). The number of ALP-positive cells increased rapidly by day 7 of culture (Figure 1b). On day 14, mineralization nodules, which were stained by von Kossa staining, appeared, but were sparse (Fig. 1c); they were more numerous and larger on day 21 (Figure 1d).

Real-time PCR for CEMP1 mRNA. The expression levels of $C E M P 1$ in the PDL cells were determined over the culture period. The expression levels of CEMP1 gradually increased from day 5 to day 14. The expression levels of CEMPl on day 7 and day 14 were approximately 1.5 and 2.3 times the level on day 5, respectively. However, the expression level of CEMPI was lower on day 21 than on day 14, and was approximately 1.8 times the level on day 5 .

\section{Discussion}

It is important to clarify the mechanisms of cementogenesis for periodontal tissue regeneration therapy. Therefore, an in vitro experimental system for investigating cementum formation is necessary. In this study, with the goal of establishing a culture system that reflects cementum formation in periodontal tissue, a primary culture of human PDL cells was prepared. We showed that human PDL tissues contain a population of cells expressing cementoblast-like phenotypes, are positive for ALP, form mineralization nodules (Figure 1), and express CEMPl (Figure 2). ALP is an important enzyme in mineralized sites, such as bone, dentin, and cementum. ALP was thought to be an early differentiation marker of osteoblasts, odontoblasts, and cementoblasts (8-11). In this culture system, there were a few ALP-positive cells on day 5, and the number of ALPpositive cells rapidly increased by day 7 . Mineralization nodules started to form by day 15 , and the nodules increased in number and size by day 21 (Figure 1). Choi et al. reported, in a primary culture system of human PDL cells that was designed in their study, that the peak expression level of ALP was reached on day 7, and that nodule mineralization started on day 15 and continued to develop to day 21 (12). These findings strongly corroborate our findings from the primary culture of human PDL cells.

The expression of CEMP1 has been shown to be limited to cementoblasts, a subpopulation of PDL cells, and to cells located around vessels in the periodontium (7). CEMP 1 was thought to be a specific marker of cementoblasts and their progenitors $(7,8)$. In this study, the expression levels of $C E M P 1$ were determined throughout the culture period, even on day 5, when there were only a few ALP-positive cells, and the expression levels of CEMP1 gradually increased from day 5 until day 14, when it reached a peak (Figure 2). Komaki et al. reported that CEMP1-positive cells were predominantly observed among ALP-positive cells in in vitro immunochemical analyses of PDL cells (8). 

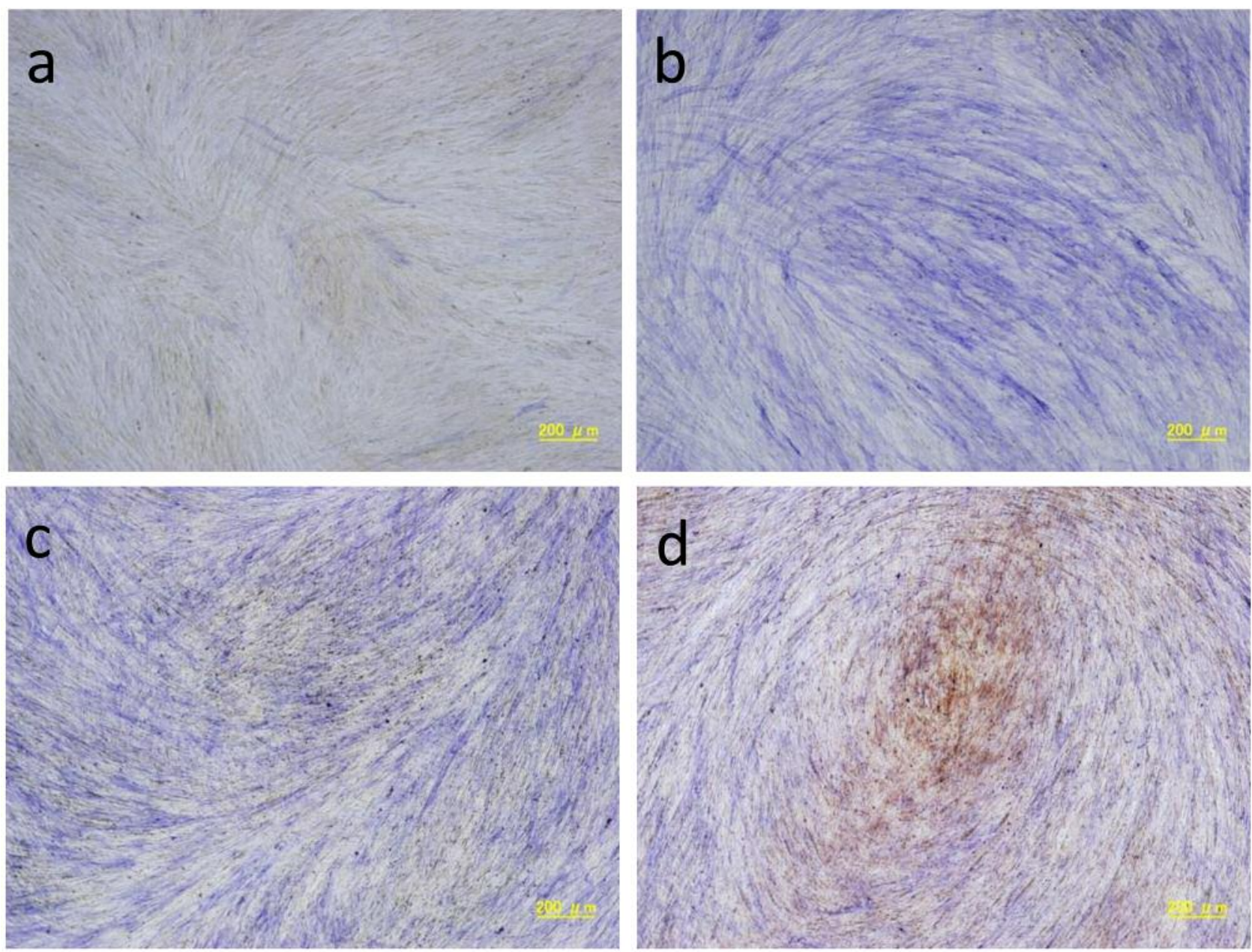

Figure 1. ALP staining and von Kossa staining of cultured PDL cells on days $5(a), 7(b), 14(c)$, and 21 (d). A few human PDL cells were positive for ALP on day $5(a)$. The number of ALP-positive cells increased rapidly by day $7(b)$. The cells formed mineralization nodules, that were stained by von Kossa staining on day $14(c)$. The mineralization nodules were larger and more numerous on day $21(d)$.

Their findings and our results indicate that the cells derived from the PDL cells in this study consisted of a heterogeneous population that included cementoblasts and/or their progenitor cells, which were positive for ALP and CEMPl, and other undifferentiated cells, which were negative for ALP on day 5. Our data suggest that almost all cells that were negative for ALP on day 5 differentiated into ALP-positive cells, and continued to differentiate into cementoblast-like cells that expressed CEMP1. In this study, the expression level of CEMPI on day 21 was decreased when compared to the level on day 14 (Figure 2). Choi et al. showed that the expression level of osteocalcin, a marker of mineralization stage, reached a peak on day 14 , and decreased by day 21 in human PDL cells in vitro (12). They also described the expression levels of c-fos, which appeared to increase prior to terminal differentiation and cell death (13). The expression level of c-fos reached a peak on day 21 in PDL cells, so they assumed that the decreased expression level of osteocalcin on day 21 was related to apoptosis during PDL cell differentiation (12). There is a possibility that the same phenomenon relating to cell death occurred in our study too, but further investigations would be necessary to clarify the mechanisms behind PDL cell death.

In conclusion, we established a PDL cell culture system in which cells differentiate into cementoblast-like cells that form mineralization nodules and express CEMPI, which is a specific marker of cementoblasts and their progenitors. Our results demonstrate that this culture system is convenient and may be useful for investigating the mechanisms of cementoblast differentiation, which would provide valuable knowledge for periodontal therapy. 


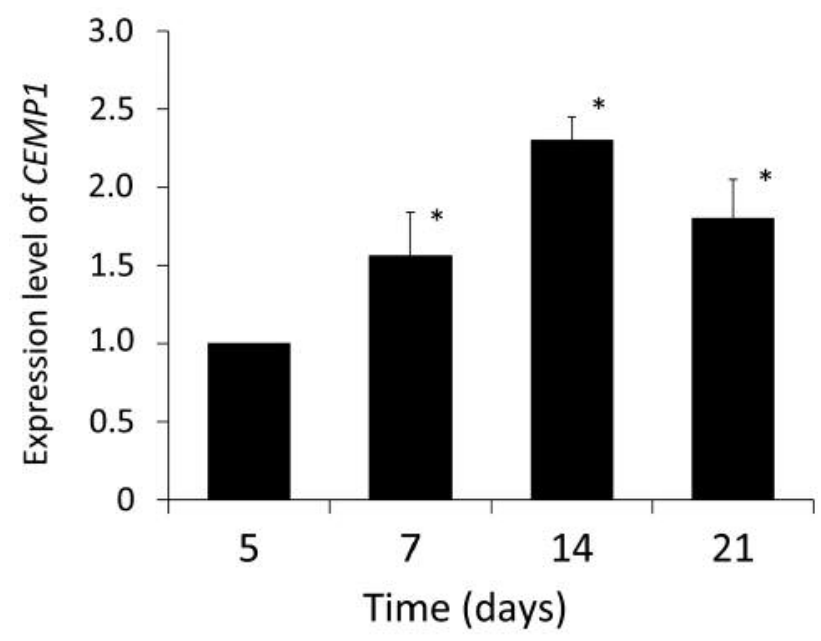

Figure 2. Changes in the mRNA expression levels of CEMP1 in the PDL cell culture over time. The expression levels shown were normalized to the expression level of GAPDH mRNA. The expression level of CEMPI gradually increased from day 5 to day 14, at which a peak was reached. The expression level of CEMP1 was decreased on day 21 in comparison to the level on day 14. Each bar represents the mean of measured values $\pm S D$ of four cultures. $* p<0.05$ when compared to the value on day 5.

\section{Conflicts of Interest}

All Authors state that they have no conflicts of interest.

\section{Authors' Contributions}

Author' role: Study design and conduct: HK and SY; Data collection: HK, TY and YK; Data analysis: HK, TY, YM and YK; Data interpretation: HK, YM, AH and SY; Drafting manuscript: HK, HS and SY; Revising manuscript: HK and SY; Approving final manuscript: HK, TY, YM, YK, AH, HS and SY. HK takes responsibility for the integrity of the data analysis.

\section{Acknowledgements}

This study was supported in part by a Grant-in-Aid for Scientific Research (16K11562) from the Ministry of Education, Science, and Culture of Japan.

\section{References}

1 Seo BM, Miura M, Gronthos S, Bartold PM, Batouli S, Brahim J, Young M, Robey PG, Wang CY and Shi S: Investigation of multipotent postnatal stem cells from human periodontal ligament. Lancet 364: 149-155, 2004. PMID: 15246727, DOI: $10.1016 / \mathrm{S} 0140-6736(04) 16627-0$
2 Gay IC, Chen $\mathrm{S}$ and MacDougall $\mathrm{M}$ : Isolation and characterization of multipotent human periodontal ligament stem cells. Orthod Craniofacial Res 10: 149-160, 2007. PMID: 17651131, DOI: 10.1111/j.1601-6343.2007.00399.x

3 Nojima N, Kobayashi M, Shionome M, Takahashi N, Suda T and Hasegawa K: Fibroblastic cells derived from bovine periodontal ligaments have the phenotypes of osteoblasts. J Periodontal Res 25: 179-185, 1990. PMID: 2163445

4 Somerman MJ, Young MF, Foster RA, Moehring JM, Imm G and Sauk JJ: Characteristics of human periodontal ligament cells in vitro. Arch Oral Biol 35: 241-247, 1990. PMID: 2161648

5 de Jong T, Bekker AD, Everts V and Smit TH: The intricate anatomy of the periodontal ligament and its development: lessons for periodontal regeneration. J Periodont Res 52: 1-10, 2017. PMID: 28635007, DOI: $10.1111 /$ jre. 12477

6 Larsson L, Decker AM, Nibali L, Pilipchuk SP, Berglundh T and Giannobile WV: Regenerative medicine for periodontal and periimplant diseases. J Dent Res 95: 255-266, 2016. PMID: 26608580, DOI: 10.1177/0022034515618887

7 Alvarez-Perez MA, Narayanan S, Zeichner-David M, Rodriguez Carmona B and Arzate H: Molecular cloning, expression and immunolocalization of a novel human cementum-derived protein (CP-23). Bone 38: 409-419, 2006. PMID: 16263347, DOI: 10.1016/j.bone.2005.09.009

8 Komaki M, Iwasaki K, Arzate H, Narayanan AS, Izumi Y and Morita I: Cementum protein 1 (CEMP1) induces a cementoblastic phenotype and reduces osteoblastic differentiation in periodontal ligament cells. J Cell Physiol 277: 649-657, 2012. PMID: 21465469, DOI: $10.1002 /$ jcp. 22770

9 Yokose S, Kadokura H, Tajima Y, Fujieda K, Katayama I, Matsuoka T and Katayama T: Establishment and characterization of a culture system for enzymatically released rat dental pulp cells. Calcif Tissue Int 66: 139-144, 2000. PMID: 10652962

10 Basdra EK and Komoposch G: Osteoblast-like properties of human periodontal ligament cells: an in vitro analysis. Eur J Orthod 19: 615-621, 1997. PMID: 9458594

11 Bonewald L: The amazing osteocyte. J Bone Miner Res 26: 229238, 2011. PMID: 21254230, DOI: $10.1002 / \mathrm{jbmr} .320$

12 Choi MX, Noh WC, Park JW, Lee JM and Suh JY: Gene expression pattern during osteogenic differentiation of human periodontal ligament cells in vitro. J Periodontal Implant Sci 41: 167-175, 2011. PMID: 21954421, DOI: 10.5051/jpis. 2011.41.4.167

13 Preston GA, Lyon TT, Yin Y, Lang JE, Solomon G, Annab L, Srinivasan DG, Alcorta DA and Barrett JC: Induction of apoptosis by c-Fos protein. Mol Cell Biol 16: 211-218, 1996. PMID: 8524298 